Article

\title{
The Evolutionary Game for Collaborative Innovation of the IoT Industry under Government Leadership in China: An IoT Infrastructure Perspective
}

\author{
Jie Hou@ and Baizhou Li *®D \\ School of Economics and Management, Harbin Engineering University, Harbin 150001, China; \\ jayholmes_hero@hrbeu.edu.cn \\ * Correspondence: lbz_hrbeu@163.com
}

Received: 5 April 2020; Accepted: 28 April 2020; Published: 1 May 2020

check for updates

\begin{abstract}
Since the United States, the European Union, China, and other countries put forward the development strategy of Internet of Things (IoT), it has been growing rapidly, leading to industrial restructuring and the sustainable development of various industries. Not only can collaborative innovation of the IoT industry accelerate R\&D efficiency and product substitution frequency of IoT infrastructure, but also it can promote the construction of smart cities, the application of Big Data and Cloud Computing, and the development of 5G. In China, government is a leader and initiator of IoT infrastructure deployment. Therefore, in this paper, we construct an evolutionary game model of the IoT industry under government leadership represented by government, telecom equipment manufacturers, and telecom operators. Then, we analyze the asymptotic stability, equilibrium stability, and evolutionary stability strategy of the model. Finally, we carry out numerical simulation to investigate the effect of eight factors and four mechanisms on evolutionary behaviors of the three parties. The results show each factor has a different influence on them. Evolutionary game model offers ideas for government and industry subjects before the collaborative innovation of IoT infrastructure deployment in practice.
\end{abstract}

Keywords: collaborative innovation; evolutionary game; government leadership; IoT infrastructure; the IoT industry

\section{Introduction}

The Internet of Things (IoT) is a global network infrastructure that connects all elements and objects through perception, identification, communication, interaction, and other ways [1,2]. It realizes the comprehensive integration of information and physical infrastructures, and has become one of the main trends affecting the development of the information technology domain. Not only does IoT improve our life standard by saving time and money, but it is applied to various fields, such as improving medical services, ensuring fire safety, increasing logistics sufficiency, controlling food safety, and building smart cities [3-5]. Besides, it is partly complementary with Big Data and Cloud Computing [6]. A large amount of diverse and unstructured information can be collected through sensors and actuators, to lay a foundation for the application and sustainable development of Big Data and Cloud Computing [7].

Nowadays, China is no longer the cheapest labor market or the leader in the high-tech domain. Although there are several achievements in aerospace, logistics, and transportation, the Chinese government still faces many risks and challenges in the implementation of the "Made in China 2025" strategy [8]. IoT, which is integrated into manufacturing process, is one of key drives of the fourth industrial revolution to deal with these difficulties effectively [9]. Weiser indicated traditional internet infrastructure would be replaced by interconnected "smart" objects in a pervasive 
computing environment [10]. Miorandi et al. further presented that it would turn into global IoT infrastructure through information sharing and transmission channels [1]. In 2017, the Chinese government put forward that it was necessary to accelerate upgrading information infrastructure and deploying IoT infrastructure for the construction of the cyber-power. IoT infrastructure deployment plays an important role in boosting domestic demand, promoting employment, and leading industrial upgrading.

Market economy in China is based on the combination of market mechanism and government mechanism. Market mechanism allocates resources through competition to realize the law of value. It has the advantages of spontaneously reducing transaction cost, balancing the relationship between supply and demand, and promoting technological innovation. However, it also has external effects, information asymmetry and other disadvantages. Due to the shortage of market mechanism, government mechanism ensures the coordinated development of economy, society, culture, and ecology through administrative decrees, financial appropriation, and other means. Telecom infrastructure, which improves the quality of life or makes government more efficient, is deployed under government leadership in China. It is not determined by enterprises or whether the business is profitable or not. This is the effect of government mechanism. For example, $4 \mathrm{G}$ technology is fully developed at present. The Chinese government put forward that it planned to make $4 \mathrm{G}$ infrastructure deployment in remote administrative villages, key boarder areas, and islands through local government coordination and policy support. It would provide important basic support for rural revitalization and poverty alleviation, and lay a sound foundation for building a moderately prosperous society in all respects. Most of the IoT products, such as smart transportation and smart water utilities, are telecom infrastructures. However, IoT technology has just been emerged for a dozen years, and is not so fully developed as 4G technology. The Chinese government strongly supports governments at all levels to deploy IoT infrastructure on a pilot. Government uses both market mechanism and government mechanism for new technologies. On one hand, market mechanism plays a fundamental role. On the other hand, government mechanism has a leading, regulating, and supporting effect on emerging industries to promote the vigorous development of new technologies. Yinchuan government issued the project that would promote the construction and application of NB-IoT, 5G, and other next-generation communication technologies of China Mobile. China has been the second largest economy in the world. However, it remains a large developing country with unbalanced regional development. Due to local economy and other factors, this could lead to a different focus for governments in regard to IoT infrastructure deployment. The government, where local economy does not go well, wants to change the current situation to overtake around the curve. It cares more about funding. On the contrary, the government with high fiscal revenue is more concerned about expected social benefit. There is another situation. The government, who receives the tasks in this respect from the top, is likely to consider both funding and expected social benefit. In addition, IoT infrastructures are complex products and systems, which are customized products and contain a great deal of innovation. Government is just a leader and initiator, who needs innovative parties to build IoT infrastructure. What kinds of partners should it choose and how should it coordinate partnerships? Further, government is also a client, who just knows about the general requirements of infrastructure, but makes nothing of the details. How should it play the role and what should it provide in the deployment of IoT infrastructure?

IoT infrastructure deployment needs technology and products above. In 2010, the Chinese government confirmed the IoT industry as a strategic emerging industry, which has novel products. It not only facilitates the rapid development of communication industry, but also drives the industrial structure adjustment and sustainable development of vertical industries and various sub-industries within the industry. Economic development in China is shifting from factor-driven or investment-driven to innovation-driven. Innovation-driven development can not only improve the overall productivity level and the quality and efficiency of economic growth, but also reduce resource consumption and improve the ecological environment to achieve 
sustainable development. The innovative development of IoT has entered the industrial explosion period of cross-border integration, integration innovation, and ecological acceleration. It combines traditional industries to change development patterns and improve industrial competitiveness, and accelerates the development of other emerging industries, such as blockchain and edge computing. Therefore, the innovative development strategy of the IoT industry is an important guarantee to build an innovative country. Furthermore, mastering industrial core technology is the key to the IoT industry. For instance, Newland chooses independent innovation on the security of 2-dimensional bar code technology for equipment cost and the initiative in the IoT industry [11]. However, in the era of internet and even IoT, with the deep integration of information and communication technology and other industries, enterprises are increasingly looking for collaborative innovation to reduce market uncertainty and high risks brought by innovation. Synergistic theory is the evolution of self-organizing system from disorder to order, i.e., the synergistic effect among subsystems generates the specific structure and function on the macro-level [12]. Collaborative innovation provides an opportunity for enterprises to explore outside. Based on internal knowledge, enterprises seek suitable partners to acquire external knowledge. They further integrate knowledge and resources, so as to explore new technologies and promote technological innovation $[13,14]$. Collaborative innovation takes various forms, e.g., network, agglomeration, and alliance [15-17]. Former researchers applied all kinds of methods to study it in various domains [18-23]. Industrial collaborative innovation has become a main factor to improve the competitiveness of the IoT industry and transform it from low-end to high-end [17]. Lu and Zhou regarded the IoT industry as an innovation system with the theory of multiply factor linkage of the innovation system, and further divided it into three subsystems, which were not only interconnected internally, but strongly interacted with each other [24]. Jiao et al. applied the evolutionary game theory to divide the collaborative innovation network development of the IoT industry in China into three stages: no dominant role, government dominant role, and alliance dominant role [25]. Shao and Lv used the symbiosis theory to study the collaborative innovation of the IoT industry led by telecom operators [26]. Yu et al. analyzed the relationship between IoT alliance and innovation with the theory of resource dependence, and found IoT alliance could promote product innovation and process innovation in IoT enterprises [27]. All in all, former researchers have applied different theories and methods to study modes and approaches of collaborative innovation about IoT. However, there are few studies on how to reach the consensus of collaborative innovation of the IoT industry under government leadership, especially involving the IoT infrastructure. Besides, the IoT industry has a huge industrial chain with unbalanced partial development. How should firms in the industry distribute benefit and what guarantees should they adopt?

Evolutionary game theory is derived from the evolution of biological population, and follows the natural laws and survival rules. It analyzes how parties with limited information play dynamic games based on established benefits [28]. Throughout all stages of dynamic game process, each party chooses a strategy by copying, learning, or inheriting, in order to make an optimal decision, which affects his/her income, respectively. The income depends more on frequencies of group strategies. Their frequencies change along with the income until they achieve the equilibrium states, respectively [29]. Evolutionary game theory is based on finite rationality, which is more in line with the reality than complete rationality [30]. Its core concepts are evolutionary stability strategy (ESS) and replicator dynamics. They can explain social and economic phenomena through evolutionary dynamic process [28]. Evolutionary game theory can analyze the evolution process in collaborative innovation of strategic emerging industries [31]. Therefore, evolutionary game theory can try to settle the matters above. That is, it is able to work out how to reach an agreement on collaborative innovation of the IoT industry under government leadership.

The first year of 5G has approached in China. 5G technology commercialization will describe a vision of global IoT with lower costs and lower energy consumption by supporting a large number of devices. Besides, $5 \mathrm{G}$ technology will be an important promoter in IoT, for the large-scale deployment of IoT infrastructure may transform the mobile value chain [32]. Meanwhile, some regions in China will 
carry out orderly frequency clearing and the withdrawal of 2G/3G network, whose base stations can be transformed to apply for IoT. Therefore, the research has certain theoretical and practical significance for IoT infrastructure innovation. Therefore, in this paper, we establish an evolutionary game model and discuss the factors, which influence collaborative innovation of the IoT industry under government leadership. We list eight factors to summarize the four mechanisms, i.e., urban planning, government intervention, distribution, and punishment, to speed up the pace of IoT infrastructure innovation.

The remainder of this paper is structured as follows. In Section 2, we analyze strategic choices of the IoT industry in collaborative innovation under government leadership. Section 3 describes the hypothesis and analysis of its evolutionary game model. In Section 4, we make numerical simulation and analysis based on the actual situation of the IoT industry under government leadership in China. The last section draws the conclusions and implications, and presents some suggestions.

\section{Strategic Choices}

\subsection{The Parties in the Evolutionary Game}

The IoT industry does not have a unified definition due to its wide range of applications. In the perspective of industrial division of labor, the IoT industry consists of manufacturing part and service part [33]. The former mainly involves the hardware, e.g., R\&D and production of products and sales of terminal equipment and network equipment. The latter mainly involves the software, e.g., software development, application integration, and platform services. IoT industry main bodies in China include terminal equipment manufacturers, telecom equipment manufacturers, network operators, software and application developers, system integrators, and operation and service providers. Terminal equipment manufacturers are enterprises who product terminal equipment. The products are mainly applied in data acquisition. Telecom equipment manufacturers are firms mainly engaged in $R \& D$, production, sales, and services of telecom equipment, especially communication modules. Telecom operators are telecommunication service companies that provide information communication channels. Software and application developers provide software and solutions for specific trade. System integrators provide manufacturers solutions through the integration of hardware and software. Operation and service providers offer various services to clients. These partners in collaborative innovation network are the key to the success of product and technology innovation [14]. Government in China often makes policies, offers services to guide industrial development, and decides whether to build infrastructure. In terms of technology and products applied to IoT base stations, in this paper we select three typical representatives: government, telecom equipment manufacturers (TEM), and telecom operators (TO).

In the national innovation system, government plays an important role in improving industry competitiveness and innovation, because the market cannot provide sufficient incentives for knowledge production [34,35]. Government intervention affects industrial $R \& D$ and innovation. It can provide enterprises resources, information, and other supports, through formulating policies and carrying out supervision, to reduce risks and uncertainties in industrial innovation. Besides, government in China is not only a leader, but also an initiator and client that has the right to make decisions to deploy IoT base stations. On one hand, government needs to promote work efficiency, increase fiscal revenue, and acquire job promotion. On the other hand, government may have no ability to make it, because of economic (fiscal revenue) or other factor. There are the two main types of governments. According to the amount of funds, the government with low fiscal revenue has two strategic choices: holding on and giving up deploying IoT base stations. The government with high fiscal revenue has only one strategic choice: holding on. It takes notice of expected social benefit. In order for it to make corresponding decisions through the threshold of expected social benefit for beneficial economic cycle and time saving, we assume that it has another strategic choice: giving up. Therefore, strategic choices of either government above can be divided into two ways: holding on and giving up deploying IoT base stations, because the government is not an innovative entity. Holding on it means the government 
takes part in collaborative innovation to make corresponding policies, carry out supervision, and provide services to the others. Giving up it means the government does not participate in collaborative innovation to offer any services to the others. In a nutshell, strategic choices of either government can be divided into two-way participation and non-participation in collaborative innovation.

Telecom industry is a technology-intensive industry with short life cycles and fast elimination rates of products. Manufacturing IoT dedicated chips, modules, and equipment needs constant innovation. IoT infrastructure also involves many vertical industries. The requirements for its positioning function, mobility management, and security mechanism are different in various application environments. As these devices and services need unified protocols and standards, collaborative innovation of the IoT industry will reduce the difficulty in R\&D. In 2016, the GSMA, together with Huawei, Vodafone, and other equipment manufacturers and operators, launched the NB-IoT Forum, aiming to expand the innovation and application of alliance members in the field of IoT, and promote the sustainable development of the IoT industrial chain. Huawei has set up several IoT Open Labs with many operators around the world, to provide services for the partners, covering public facilities, Internet of cars, industrial manufacturing, and other fields. Therefore, TEM and TO are innovative subjects, who play a key role in R\&D and product substitution of IoT infrastructure, like NB-IoT network, whereas TEM and TO are the enterprises for profit, they will give up collaborative innovation if there are no profit to earn. Therefore, the strategic selections of TEM and TO can be divided into two-way participation and non-participation, respectively. TEM within collaborative innovation can perform product and technology innovation. TEM outside it can utilize the other's technology to innovate. The performance of TO is the same as that of TEM.

\subsection{The Game among the Parties}

After the above analysis, there are the three typical representatives in the evolutionary game model: government, TEM, and TO. Each party has two different strategic choice: participation and non-participation, respectively. The game among the parties are as follows.

Government is an initiator and client of the IoT infrastructure deployment. It just wants the quantity of IoT base stations that meet basic requirements, and provides reasonable subsidy. However, these may increase innovation risks for TEM/TO. Besides, the government may not, or postpone to offer, necessary help, such as land capacity map or other agreement. It increases difficulty of innovation for TEM/TO. TEM/TO will weigh the gains and losses to choose the corresponding strategic choice. In turn, TEM/TO demands the subsidy that may be over the government's budget. Or IoT infrastructure may not bring corresponding social benefit. The government will think twice.

TEM and TO are innovative parties. In China, TO are state-owned enterprises, who have a huge scale and relatively stable operating income. Most TEM are small and medium-sized enterprises, who face a great risk during innovation. Therefore, how to distribute government subsidy and benefit will have an impact on their strategic choices, respectively.

In conclusion, TEM and TO are producers, who follow the market mechanism. They are in charge of R\&D and innovation of products through technical cooperation and coordination. Government takes advantage of government mechanism to assists producers to make up for the deficiencies of market mechanism. In this way, the collaborative innovation system is constructed with enterprises as the innovative subjects and government as the impetus. Figure 1 displays the evolutionary game diagram of the government, TEM, and TO in collaborative innovation. 


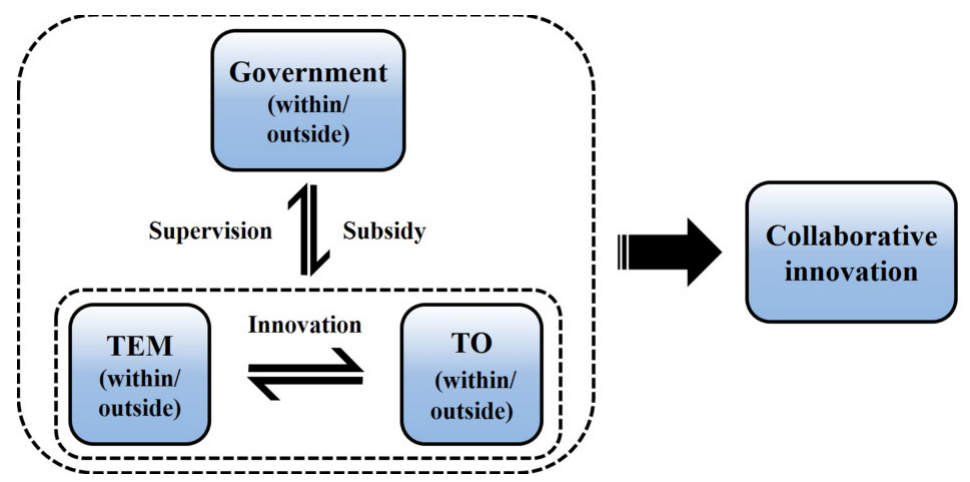

Figure 1. The evolutionary game diagram of the government, telecom equipment manufacturers (TEM), and telecom operators (TO) in collaborative innovation.

\section{Model}

As the main bodies of the collaborative innovation system, the government, TEM, and TO follow market mechanism and government mechanism. In the case of information opacity, there will be various shortcomings, such as profit seeking or policy failure. Evolutionary game theory helps participants understand the dynamic process of group evolution and explain why and how the group achieves the state. It has no need for complete rationality and information transparency. Meanwhile, governments at all levels, TEM, and TO are numerous in China and they can continuously learn. Therefore, an evolutionary game model can analyze the evolution process of collaborative innovation of the IoT industry under government leadership.

\subsection{Assumption and Payoff Matrix}

In the evolutionary game model, we hypothesize that the government, TEM, and TO are regarded as a system in the environment with effective government and efficient market. TEM and TO are in charge of product development and technology innovation, which are applied in IoT infrastructure. The government takes care of subsidy, supervision, and other services. The three parties are all limited rational and have found the optimal strategic solution through multiple games. Table 1 shows the parameter assumptions of cost, benefit, subsidy, and penalty.

The three parties have two kinds of strategic selections in the model, respectively. We set $x(0<x<1), y(0<y<1)$, and $z(0<z<1)$ as the frequencies of the government, TEM, and TO going in for collaborative innovation, respectively. Correspondingly, the frequencies of the government, TEM, and TO dropping out of collaborative innovation are $1-x, 1-y$, and $1-z$, respectively. Table 2 demonstrates the pay-off matrix of the evolutionary game for collaborative innovation of the IoT industry under government leadership.

Table 1. Parameter assumption.

\begin{tabular}{lll}
\hline Assumption & \multicolumn{1}{c}{ Parameter } & \multicolumn{1}{c}{ Description } \\
\hline \multirow{3}{*}{ Cost } & \multicolumn{1}{c}{$C$} & The cost of the government in collaborative innovation \\
\cline { 2 - 3 } & \multicolumn{1}{c}{$\alpha(0<\alpha<1)$} & The total cost of TEM and TO in collaborative innovation \\
\hline \multirow{3}{*}{ Benefit } & $R_{G}$ & The reduction of $C$ in tripartite collaborative innovation \\
\cline { 2 - 3 } & $R_{1}(1<\gamma)$ & The social benefit in collaborative innovation only containing TEM and TO \\
\cline { 2 - 3 } & $R_{2}$ & The benefit of TEM before collaborative innovation \\
\cline { 2 - 3 } & $n(0<n)$ & The benefit of TO before collaborative innovation \\
\hline
\end{tabular}


Table 1. Cont

\begin{tabular}{|c|c|c|}
\hline Assumption & Parameter & Description \\
\hline \multirow{4}{*}{ Benefit } & $\Delta R$ & The benefit of an IoT base station \\
\hline & $\beta(0<\beta<1)$ & $\begin{array}{l}\text { The proportion coefficient of the benefit of TEM in the total benefit of TEM } \\
\text { and TO in collaborative innovation }\end{array}$ \\
\hline & $R_{M}$ & $\begin{array}{l}\text { The benefit of TO outside collaborative innovation, who utilizes some } \\
\text { technology from TEM within it }\end{array}$ \\
\hline & $R_{O}$ & $\begin{array}{l}\text { The benefit of TEM outside collaborative innovation, who utilizes some } \\
\text { technology from TO within it }\end{array}$ \\
\hline \multirow{5}{*}{$\begin{array}{l}\text { Subsidy/ } \\
\text { Penalty }\end{array}$} & G & The amount of government subsidy in collaborative innovation \\
\hline & $\delta(0 \leq \delta \leq 1)$ & The proportion coefficient of the subsidy to TEM in $G$ \\
\hline & $\theta(0<\theta<1)$ & $\begin{array}{l}\text { The proportion coefficient of the compensation of TEM within collaborative } \\
\text { innovation by TO outside it in } C\end{array}$ \\
\hline & $\rho(0<\rho<1)$ & $\begin{array}{l}\text { The proportion coefficient of the compensation of TO within collaborative } \\
\text { innovation by TEM outside it in } C\end{array}$ \\
\hline & $2 G_{L} / G_{L}$ & $\begin{array}{l}\text { The compensation of the government by TEM and/or TO outside } \\
\text { collaborative innovation }\end{array}$ \\
\hline
\end{tabular}

Table 2. The pay-off matrix of the evolutionary game model.

\begin{tabular}{|c|c|c|c|c|c|}
\hline \multicolumn{2}{|c|}{ Government } & \multicolumn{2}{|c|}{ Within $(x)$} & \multicolumn{2}{|c|}{ Outside $(1-x)$} \\
\hline \multirow{6}{*}{ TEM } & \multirow{3}{*}{$\begin{array}{l}\text { Within } \\
(y)\end{array}$} & $\gamma R_{G}-C_{G}-G$ & $G_{L}-\delta G-C_{G}$ & $R_{G}$ & 0 , \\
\hline & & $\begin{array}{l}R_{1}+n \beta \Delta R+\delta G- \\
\alpha(C-S)\end{array}$ & $\begin{array}{l}R_{1}+\theta C+\delta G- \\
\alpha(C-S)\end{array}$ & $R_{1}+n \beta \Delta R-\alpha C$ & $R_{1}+\theta C-\alpha C$ \\
\hline & & $\begin{array}{l}R_{2}+n(1-\beta) \Delta R+(1- \\
\delta) G-(1-\alpha)(C-S)\end{array}$ & $R_{2}+R_{M}-\theta C-G_{L}$ & $\begin{array}{l}R_{2}+n(1-\beta) \Delta R- \\
(1-\alpha) C\end{array}$ & $R_{2}+R_{M}-\theta C$ \\
\hline & \multirow{3}{*}{$\begin{array}{l}\text { Outside } \\
(1-y)\end{array}$} & $G_{L}-(1-\delta) G-C_{G}$ & $2 G_{L}-C_{G}$ & 0 & 0 , \\
\hline & & $R_{1}+R_{O}-\rho C-G_{L}$ & $R_{1}-G_{L}$ & $R_{1}+R_{O}-\rho C$ & $R_{1}$ \\
\hline & & $\begin{array}{l}R_{2}+\rho C+(1-\delta) G- \\
(1-\alpha)(C-S)\end{array}$ & $R_{2}-G_{L}$ & $R_{2}+\rho C-(1-\alpha) C$ & $R_{2}$ \\
\hline \multicolumn{2}{|c|}{ TO } & Within $(z)$ & Outside $(1-z)$ & Within $(z)$ & Outside $(1-z)$ \\
\hline
\end{tabular}

\subsection{Expected Revenue Function and Replication Dynamic System}

According to Table 2, the participation, non-participation, and average expected revenue functions of the government are $U_{G 1}, U_{G 2}$, and $\bar{U}_{G}$, respectively. We can obtain

$$
\begin{aligned}
& U_{G 1}=y z\left(\gamma R_{G}-C_{G}-G\right)+y(1-z)\left(G_{L}-\delta G-C_{G}\right) \\
& +(1-y) z\left[G_{L}-(1-\delta) G-C_{G}\right]+(1-y)(1-z)\left(2 G_{L}-C_{G}\right) \text {. } \\
& U_{G 2}=y z R_{G} . \\
& \bar{U}_{G}=x U_{G 1}+(1-x) U_{G 2} \text {. }
\end{aligned}
$$

Similarly, the participation, non-participation, and average expected revenue functions of TEM are $U_{M 1}, U_{M 2}$, and $\bar{U}_{M}$, respectively. The participation, non-participation, and average expected revenue functions of TO are $U_{O 1}, U_{O 2}$, and $\bar{U}_{O}$, respectively. We can obtain

$$
\begin{aligned}
U_{M 1}= & x z\left[R_{1}+n \beta \Delta R+\delta G-\alpha(C-S)\right]+x(1-z)\left[R_{1}+\theta C+\rho G-\alpha(C-S)\right] \\
& +(1-x) z\left(R_{1}+n \beta \Delta R-\alpha C\right)+(1-x)(1-z)\left(R_{1}+\theta C-\alpha C\right) .
\end{aligned}
$$




$$
\begin{aligned}
& U_{M 2}=x z\left(R_{1}+R_{O}-\rho C-G_{L}\right)+x(1-z)\left(R_{1}-G_{L}\right) \\
& +(1-x) z\left(R_{1}+R_{O}-\rho C\right)+(1-x)(1-z) R_{1} \text {. } \\
& \bar{U}_{M}=y U_{M 1}+(1-y) U_{M 2} \text {. } \\
& U_{O 1}=x y\left[R_{2}+n(1-\beta) \Delta R+(1-\delta) G-(1-\alpha)(C-S)\right]+(1-x)(1-y)\left[R_{2}+\rho C-(1-\alpha) C\right] \\
& +x(1-y)\left[R_{2}+\rho C+(1-\delta) G-(1-\alpha)(C-S)\right]+(1-x) y\left[R_{2}+n(1-\beta) \Delta R-(1-\alpha) C\right] . \\
& U_{O 2}=x y\left(R_{2}+R_{M}-\theta C-G_{L}\right)+x(1-y)\left(R_{2}-G_{L}\right) \\
& +(1-x) y\left(R_{2}+R_{M}-\theta C\right)+(1-x)(1-z) R_{2} \text {. } \\
& \bar{U}_{O}=z U_{O 1}+(1-z) U_{O 2} \text {. }
\end{aligned}
$$

Replication dynamic equations of the government, TEM, and TO are $F_{G}(x), F_{M}(y)$, and $F_{O}(z)$, respectively. We depict the objective functions as

$$
\begin{gathered}
F_{G}(x)=d x / d t=x\left(U_{G 1}-\bar{U}_{G}\right) . \\
F_{M}(y)=d y / d t=y\left(U_{M 1}-\bar{U}_{M}\right) . \\
F_{O}(z)=d z / d t=z\left(U_{O 1}-\bar{U}_{O}\right) .
\end{gathered}
$$

Equations (1) and (3) are combined to obtain Equation (10). Equations (4) and (6) are combined to obtain Equation (11). Equation (12) is obtained in the combination of Equations (7) and (9). Therefore, we obtain the replication dynamic system of the government, TEM, and TO in the combination of Equations (10)-(12), which can be depicted as

$$
\left\{\begin{array}{l}
F_{G}(x)=x(1-x) h(y, z) \\
F_{M}(y)=y(1-y) g(x, z) \\
F_{O}(z)=z(1-z) f(x, y)
\end{array}\right.
$$

where $f(x, y), g(x, z)$, and $h(y, z)$ are specific as

$$
\begin{gathered}
f(x, y)=x\left[(1-\delta) G+G_{L}+(1-\alpha) S\right]+y\left[n(1-\beta) \Delta R-R_{M}\right]-[(1-\alpha-\rho)+y(\rho-\theta)] C . \\
g(x, z)=x\left(\delta G+G_{L}+\alpha S\right)+z\left(n \beta \Delta R-R_{O}\right)-[(\alpha-\theta)+z(\theta-\rho)] C . \\
h(y, z)=y z(\gamma-1) R_{G}+(2-y-z) G_{L}-[y \delta+z(1-\delta)] G-C_{G} .
\end{gathered}
$$

\subsection{Equilibrium Stability and ESS}

According to system Equation (13), we have sixteen equilibrium points when $F_{G}(x)=F_{M}(y)=$ $F_{O}(z)=0$. There are eight special equilibrium points, i.e., $E_{1}=(0,0,0), E_{2}=(0,0,1), E_{3}=(0,1,0)$, $E_{4}=(0,1,1), E_{5}=(1,0,0), E_{6}=(1,0,1), E_{7}=(1,1,0)$, and $E_{8}=(1,1,1)$. They constitute the boundary of the solution domain. In addition, there may be eight local equilibrium points, that is, $E_{9}=\left(x_{9}, y_{9}, z_{9}\right), E_{10}=\left(x_{10}, y_{10}, z_{10}\right), E_{11}=\left(0, y_{11}, z_{11}\right), E_{12}=\left(1, y_{12}, z_{12}\right), E_{13}=\left(x_{13}, 0, z_{13}\right), E_{14}=$ $\left(x_{14}, 1, z_{14}\right), E_{15}=\left(x_{15}, y_{15}, 0\right)$, and $E_{16}=\left(x_{16}, y_{16}, 1\right)$.

We analyze equilibrium stability and ESS of these sixteen equilibrium points. According to system Equation (13), the derivatives of $F_{G}(x), F_{M}(y)$, and $F_{O}(z)$ are $F_{G}^{\prime}(x), F_{M}^{\prime}(y)$, and $F_{O}^{\prime}(z)$, respectively. We can obtain

$$
\left\{\begin{array}{l}
F_{G}^{\prime}(x)=(1-2 x) h(y, z) \\
F_{M}^{\prime}(y)=(1-2 y) g(x, z) . \\
F_{O}^{\prime}(z)=(1-2 z) f(x, y)
\end{array}\right.
$$


When $E\left(x^{*}, y^{*}, z^{*}\right)$ equals $E_{9}$ to $E_{16}$, respectively, at least two of $F_{G}^{\prime}(x), F_{M}^{\prime}(y)$, and $F_{O}^{\prime}(z)$ are equal to zero. The linearization theorem cannot apply in this situation. According to its phase diagram, the system approaches the equilibrium point from the left of $x^{*}, y^{*}$, or $z^{*}$, but escapes the equilibrium point to the right of $x^{*}, y^{*}$, or $z^{*}$, and vice versa. As a result, $E\left(x^{*}, y^{*}, z^{*}\right)$ is not asymptotically stable. That is, $E_{9}$ to $E_{16}$ is neither asymptotically stable, nor the ESS, respectively [36]. When $E\left(x^{*}, y^{*}, z^{*}\right)$ equals $E_{1}$ to $E_{8}$, respectively, none of $F_{G}^{\prime}(x), F_{M}^{\prime}(y)$, and $F_{O}^{\prime}(z)$ equal zero. According to the linearization theorem, $E\left(x^{*}, y^{*}, z^{*}\right)$ is asymptotically stable, i.e., $E_{1}$ to $E_{8}$ is asymptotically stable, respectively [36].

According to the linearization theorem and the method proposed by Friedman, if both an equilibrium point and its linearization are asymptotically stable, no eigenvalue of the Jacobian matrix has strictly positive real part [28,36]. We can obtain the ESS of system Equation (13) after local stability analysis of the system Jacobian matrix, which can be expanded

$$
J=\left[\begin{array}{ccc}
F_{G}^{\prime}(x) & x(1-x) h_{y}^{\prime}(y, z) & x(1-x) h_{z}^{\prime}(y, z) \\
y(1-y) g_{x}^{\prime}(x, z) & F_{M}^{\prime}(y) & y(1-y) g_{z}^{\prime}(x, z) \\
z(1-z) f_{x}^{\prime}(x, y) & z(1-z) f_{y}^{\prime}(x, y) & F_{O}^{\prime}(z)
\end{array}\right]
$$

where $h_{y}^{\prime}(y, z)$ and $h_{z}^{\prime}(y, z)$ are partial derivatives of $h(y, z), g_{x}^{\prime}(x, z)$ and $g_{z}^{\prime}(x, z)$ are partial derivatives of $g(x, z)$, and $f_{x}^{\prime}(x, y)$ and $f_{y}^{\prime}(x, y)$ are partial derivatives of $f(x, y)$.

To satisfy the actual situation in collaborative innovation of the IoT industry under government leadership, further assumptions are required.

- The difference between the social benefit in collaborative innovation of the three parties and that of TEM and TO should be greater than the sum of the cost and subsidy of the government. The benefit of TEM/TO in tripartite collaborative innovation should greater than the sum of its own cost and the benefit of itself outside collaborative innovation. That is, $(\gamma-1) R_{G}>G+C_{G}$, $n \beta \Delta R>\alpha C+R_{O}$, and $n(1-\beta) \Delta R>(1-\alpha) C+R_{M}$.

- The cost of the government in collaborative innovation should be greater than the biggest compensation of itself. The cost of TEM/TO should be greater than the compensation of itself. Namely, $C_{G}>2 G_{L}, \alpha>\theta$, and $1-\alpha>\rho$.

We put $E_{1}$ to $E_{8}$ in Equation (18) to calculate the corresponding eigenvalue and stability of each equilibrium point, respectively. Table 3 shows that $E_{1}$ and $E_{8}$ are the ESS of the replication dynamic system. In other words, the government, TEM, and TO have two choices. Either all or none can cooperate in innovation.

Table 3. Eigenvalue and stability of each equilibrium point.

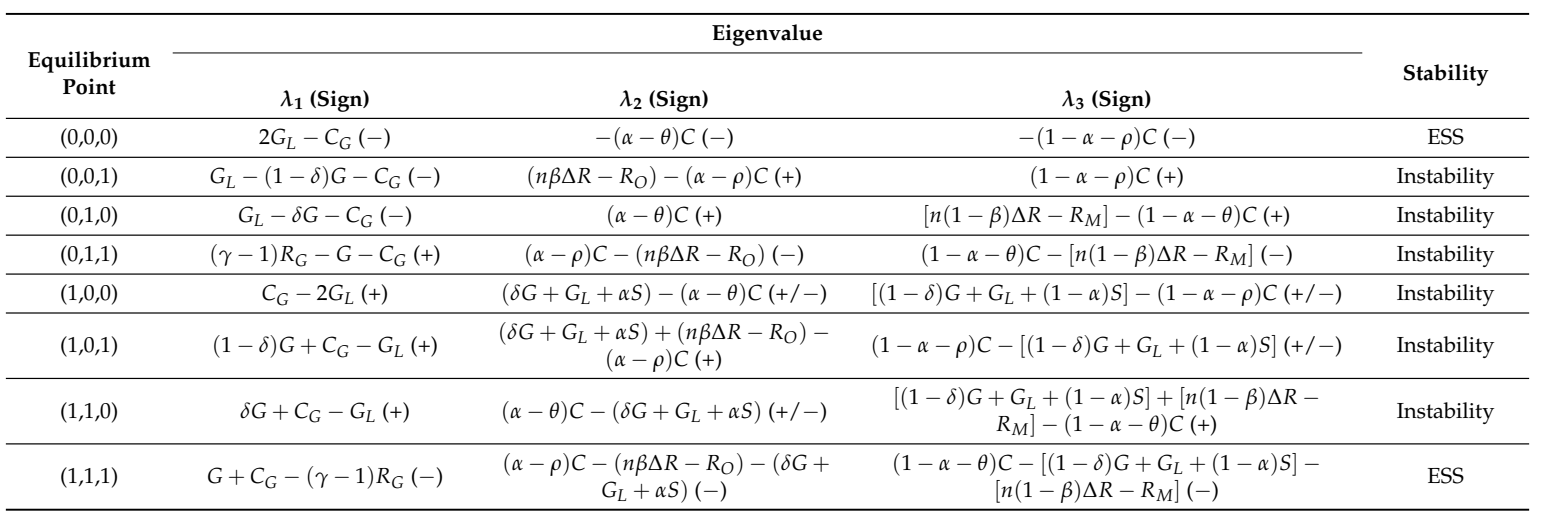

\subsection{Asymptotic Stability}

In order to study which factor affects the ESS of the replication dynamic system, we carry out asymptotic stability analysis of the parties. According to the evolutionary game theory and system 
Equation (17), when $F_{G}^{\prime}\left(x^{*}\right)<0, F_{M}^{\prime}\left(y^{*}\right)<0$, and $F_{O}^{\prime}\left(z^{*}\right)<0, E\left(x^{*}, y^{*}, z^{*}\right)$ will be the ESS in collaborative innovation of the IoT industry under government leadership [36].

The asymptotic stability analysis of the government: According to system Equation (13), when $h(y, z)=0, F_{G}(x) \equiv 0 . x$ does not change over time. $x=0$ and $x=1$ are the two stable components of $x$ above. When $h(y, z)>0, F_{G}^{\prime}(0)>0$ and $F_{G}^{\prime}(1)<0 . x=1$ is the balance component of government's strategic selection. That is, if the sum of the social benefit in collaborative innovation of the three parties and the compensation of the government is greater than the sum of the cost and subsidy of the government and the social benefit without the government, the government will take part in collaborative innovation. On the contrary, when $h(y, z)<0, x=0$ is the balance component. The government will not take part in it. Figure $2 a-c$ show the dynamic trends and stability of the government under different conditions.

The asymptotic stability analysis of TEM: According to system Equation (13), when $g(x, z)=0$, $F_{M}(y) \equiv 0 . y$ does not change over time. $y=0$ and $y=1$ are the two stable components of $y$. When $g(x, z)>0, F_{M}^{\prime}(0)>0$ and $F_{M}^{\prime}(1)<0 . y=1$ is the balance component of TEM's strategic selection. That is, if the sum of the benefit and the guarantee of TEM in tripartite collaborative innovation is greater than the sum of its own cost and the benefit of itself outside collaborative innovation, TEM will join collaborative innovation. On the contrary, when $g(x, z)<0, y=0$ is the balance component. TEM will not join it. Figure $2 \mathrm{~d}-\mathrm{f}$ demonstrate the dynamic trends and stability of TEM under different conditions.

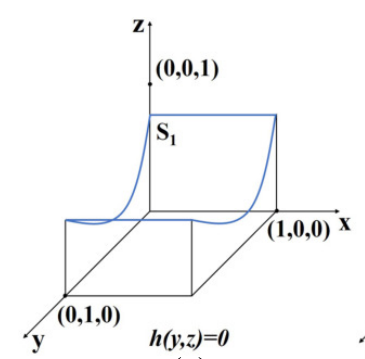

(a)

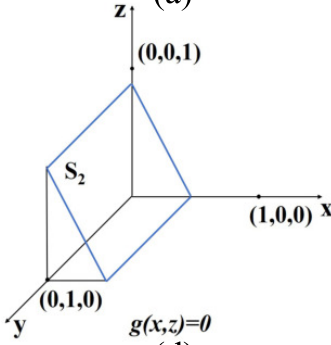

(d)

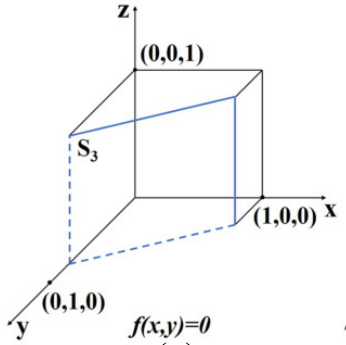

(g)

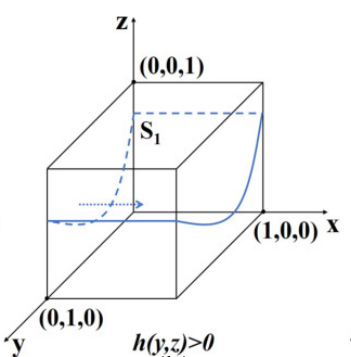

(b)

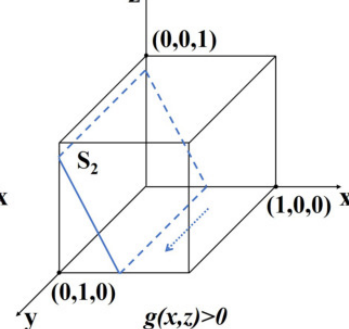

(e)

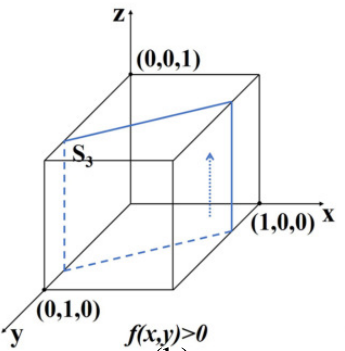

(h)

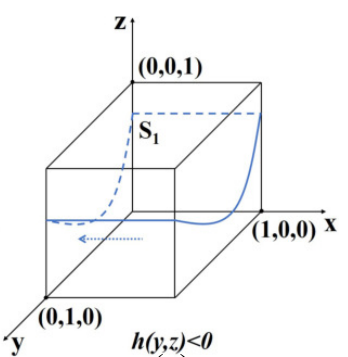

(c)

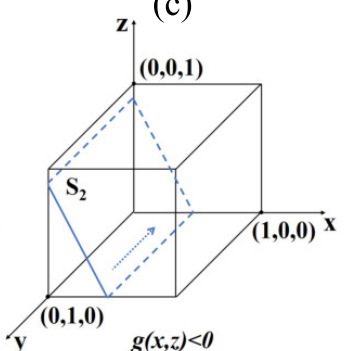

(f)

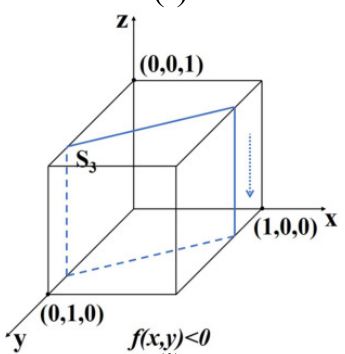

(i)

Figure 2. The dynamic trends and stability of the government $(\mathbf{a}-\mathbf{c})$, TEM $(\mathbf{d}-\mathbf{f})$, and TO $(\mathbf{g}-\mathbf{i})$ under different conditions.

The asymptotic stability analysis of TEM: According to system Equation (13), when $f(x, y)=0$, $F_{O}(z) \equiv 0 . \quad z$ does not change over time. $z=0$ and $z=1$ are the two stable components of $z$. When $f(x, y)>0, F_{O}^{\prime}(0)>0$ and $F_{O}^{\prime}(1)<0 . z=1$ is the balance component of TO's strategic selection. That is, if the sum of the benefit and the guarantee of TO in tripartite collaborative innovation is greater 
than the sum of its own cost and the benefit of itself outside collaborative innovation, TO will go in for collaborative innovation. On the contrary, when $f(x, y)<0, z=0$ is the balance component. TO will not go in for it. Figure $2 \mathrm{~g}-\mathrm{i}$ display the dynamic trends and stability of TO under different conditions.

According to different results obtained above, if the government, TEM, and TO want to participate in collaborative innovation at the same time, all Equations (14)-(16) are greater than zero. Therefore, we list eight factors to come up with the four mechanisms of urban planning, government intervention, distribution, and punishment to enhance collaborative innovation.

\section{Numerical Simulation}

In order to show dynamic evolution trends of the government, TEM, and TO under certain conditions, we use Matlab 2018b to conduct numerical simulation of the three parties. Table 4 shows the parameters assigned by the reality of the IoT industry in China and the assumptions above. A unit is a million yuan.

Table 4. Parameter assignment.

\begin{tabular}{cccccccccc}
\hline Cost & & $C_{G}$ & & $C$ & & $\alpha$ & & $S$ & \\
Value & & 18 & & 100 & & 0.5 & & 12 & \\
\hline Profit & $R_{G}$ & $\gamma$ & $R_{1}$ & $R_{2}$ & $n$ & $\Delta R$ & $\beta$ & $R_{M}$ & $R_{O}$ \\
Value & 27 & 4 & 45 & 54 & 40 & 5 & 0.5 & 40 & 48 \\
\hline $\begin{array}{c}\text { Subsidy/Penalty } \\
\text { Value }\end{array}$ & $G$ & & $\delta$ & & $\theta$ & & $\rho$ & & $G_{L}$ \\
\hline
\end{tabular}

Figure 3 displays the influence of different participation intentions of the government, TEM, and TO on each evolutionary behavior. All values of $x, y$, and $z$ range from 0 to 1 with the step of 0.1 , respectively. The points on the curves mean different participation intentions of the three parties in the system. Further, the curves signify the evolution paths of the points on them. The arrow direction represents the evolution direction of the system. In other words, different participation intentions of the three parties in the system have an impact on the consensus on collaborative innovation of the IoT industry under government leadership. Figure 3 shows that almost all lines converge to two points, $(0,0,0)$ and $(1,1,1)$. It is consistent with the discussion above. The stronger the participation intentions of the three parties are, the more likely they are to promote collaborative innovation. In this paper, we initially set the participation and non-participation of the three parties in collaborative innovation as 0.5 , respectively. Then, according to the above parameters, we discuss the eight factors and four mechanisms.

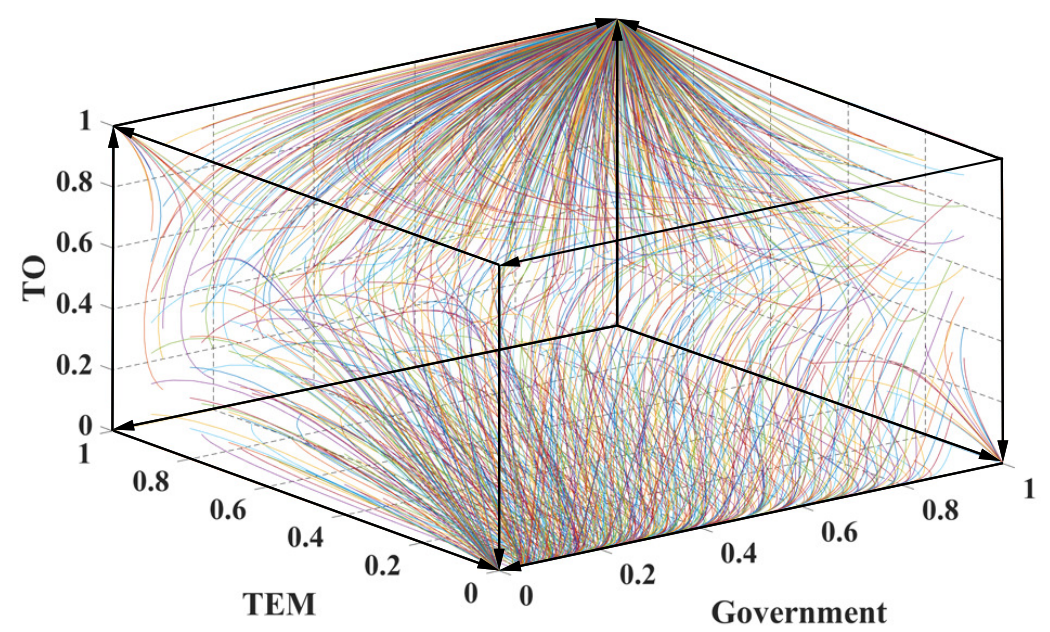

Figure 3. The influence of different participation willingness of the government, TEM, and TO on each evolutionary behavior, and the arrow direction represents the evolution direction of the system. 


\subsection{Urban Planning}

The rapid growth of urban population density requires a lot of infrastructure to meet the demand of residents. Urban planning and design explores the innovation of urban planning in practice, in order to balance the requirements of environment, aesthetics, comfort, and safety. It provides ecosystem services to realize social value and affect national sustainable development [37]. In recent years, the development and expansion of the IoT industry has provided commercial prospect for building smart cities, and gradually replaced traditional systems with intelligent and renewable urban system, to achieve a more effective urban economic, social, and environmental planning and management [38,39]. In this paper, we use $n$ and $\gamma$ as representatives of urban planning. Figure 4 a shows the influence of $n$ on the evolutionary behaviors of the three parties. The value of $n$ ranges from 1 to 100 with the step of 1 , and the arrow direction represents an increase in value. As $n$ keeps increasing, all of the three parties evolve from non-participation to participation in collaborative innovation gradually. According to engineering, Top view is an orthographic projection of the object from above to below. Front view is an orthographic projection of the object from front to back. Side view is an orthographic projection of the object from left to right. In front and top views, as $n$ keeps increasing, the slope of the curve near the central point changes from large to small to large. It indicates the $n$ has a more severe impact on the participation willingness of TEM and TO than that of the government. In side view, the graph is like of a linear shape. It indicates the $n$ has a similar impact on the participation intentions of TEM and TO. Figure $4 \mathrm{~b}$ displays the influence of $\gamma$ on the evolutionary behaviors of the three parties. The value of $\gamma$ ranges from 1 to 10 with the step of 0.1 , and the arrow direction represents an increase in value. As $\gamma$ keeps increasing, all of the three parties evolve from non-participation to participation in collaborative innovation gradually. In front and top views, as $\gamma$ keeps increasing, the slope of the curve near the central point changes from small to large to small. It indicates the $\gamma$ has a more severe impact on the participation willingness of the government than that of TEM and TO. In side view, the graph is also resembles a linear shape. It indicates the $\gamma$ has a similar impact on the participation intentions of TEM and TO. In conclusion, $n$ has a lower limit. It directly affects the participation willingness of TEM and TO, and then drives that of the government to change together in the evolutionary game process. In other words, the increasing number of IoT base stations will promote the innovative parties to reach the consensus of collaborative innovation. $\gamma$ also has a lower limit. It directly affects the participation willingness of the government, and then drives that of TEM and TO to change together. Further, the increasing expected social benefit will accelerate the government to reach the agreement of collaborative innovation. Both $n$ and $\gamma$ essentially follow market mechanism.
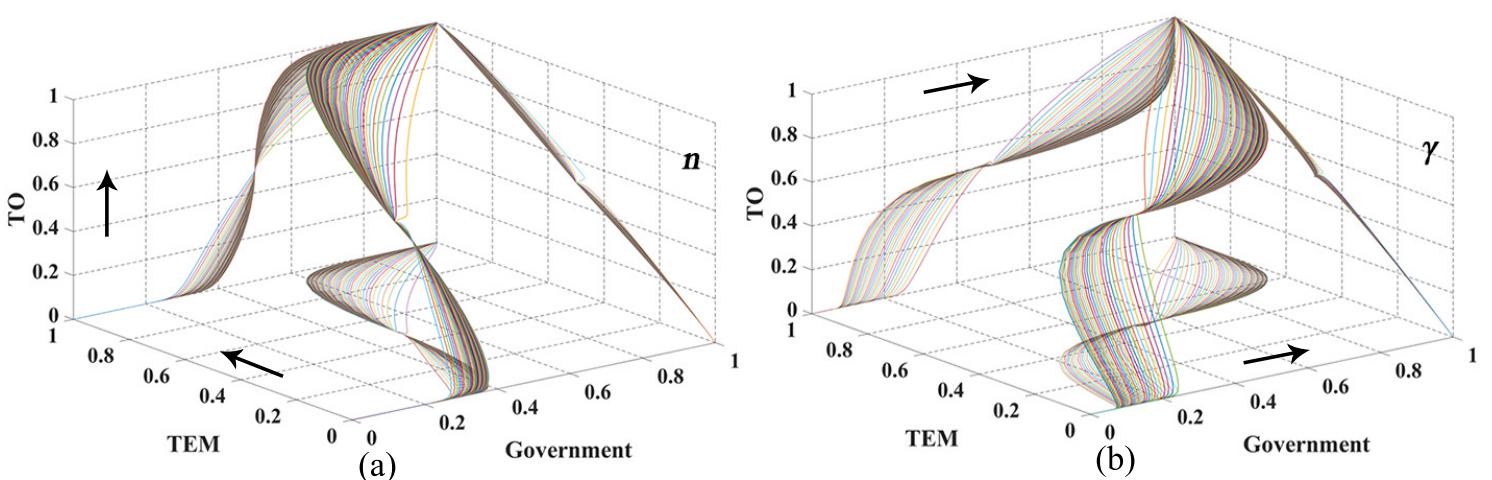

Figure 4. The influence of $n(\mathbf{a})$ and $\gamma(\mathbf{b})$ on the evolutionary behaviors of the three parties, and the arrow direction represents an increase in value.

Yingtan municipal government has signed cooperation agreements with Huawei and CMCC, ZTE and China Telecom, and Huawei and China Unicom, respectively. They have carried out the deep cooperation in NB-IoT industrial ecology, intelligent application, Cloud Computing, and other fields to build "wisdom city" by constructing the six-tier architecture of the IoT industry development. 
In reality, IoT, like NB-IoT, belongs to the cellular network and covers a certain area. The area under the jurisdiction of local government is instant but the population distribution is different. The deployment (number and location) of IoT base stations will affect the return on investment of telecom enterprises directly. At the beginning, the greater the number of base stations is, the more that return on investment of telecom enterprises increases, like the hypothesis content above. However, when the number continues to increase at a relative saturation, the marginal profit of them will be reduced significantly. Besides, the deployment of IoT base stations is customized product, which faces a lot of risk. The cost of a base station is generally fixed. The more the number of base stations is, the lower innovative parties' marginal cost and innovation risk are. Government improves the social benefit by expanding the business and application of IoT. These can affect work efficiency, fiscal revenue, and job promotion of local government. The IoT industry has a long industrial chain, which covers most of industries. IoT infrastructure is applied to communication, management, and computing requirements for building smart city from network, cloud, and data, respectively [40]. For example, IoT can be applied to intelligent transportation, intelligent building, and other public fields through Cloud Computing and Big Data [41,42]. It can make full use of social resource allocation, and accelerate the pace of building smart cities and creating an intelligent society [43,44]. However, the deployment of IoT base stations takes a lot of time. Different businesses and applications of IoT bring about different social benefits to government. The government with high fiscal revenue is able to choose the corresponding IoT infrastructure at first, which brings about the highest social benefit. This finally creates a beneficial economic cycle. Otherwise, there are several projects to be constructed simultaneously for time saving. Therefore, government and telecom enterprises should coordinate the urban planning and design of IoT infrastructure, and fully consider its $5 \mathrm{~W} 1 \mathrm{H}$ problem. Creating incentive targets with rich benefits and full motivation lays a solid economic foundation for collaborative innovation of the IoT industry under government leadership.

\subsection{Government Intervention}

The IoT industry is a strategic emerging industry. Government identifies innovation tasks and challenges through innovation institutional environment, industrialization capacity, and technical characteristics. It further affects industrial R\&D by means of policy, financial, and other intervention tools $[45,46]$. Most of government intervention take on directive intervention and facilitative intervention. Directive intervention includes R\&D subsidies, tax incentives, and public procurement, and facilitative intervention involves infrastructure construction, professional services, and regulation [34]. Each kind of government intervention has a different role and potential [47]. In this paper, we use $G$ and $S$ as representatives of government intervention. Figure 5 a demonstrates the influence of $G$ on the evolutionary behaviors of the three parties. The value of $G$ ranges from 0 to 100 with the step of 1 , and the arrow direction represents an increase in value. As $G$ keeps increasing, all the three parties evolve from non-participation to participation to non-participation in collaborative innovation gradually. In front and top views, we can see the intersections of the curves and the axis representing the participation intention of the government. When $G$ is very small, TEM and TO give up first, resulting in the government followed to abandon collaborative innovation. With the constant increase in $G$, after it reaches the first critical value, all of the three parties begin to take part. However, the government is less willing to go at this time. It is TEM and TO that drive the government to carry out collaborative innovation. With the further increase of $G$ to the second critical value, the government gives up first, leading to the breakdown of the tripartite relationship. In side view, the graph also shows a linear shape, indicating the $G$ has a similar influence on the participation willingness of TEM and TO. Figure $5 \mathrm{~b}$ shows the influence of $S$ on the evolutionary behaviors of the three parties. The value of $S$ ranges from 0 to 20 with the step of 0.2 , and the arrow direction represents an increase in value. As $S$ keeps increasing, all of the three parties evolve from non-participation to participation in collaborative innovation gradually. In front, top, and side views, there is no significant difference in the influence of the $S$ on the participation intentions of the three parties. To sum up, $G$ can only be in a range. It can directly affect the participation intentions of the 
government, TEM, and TO in the evolutionary game process. In other words, government subsidy within a reasonable range will promote the three parties to reach the consensus of collaborative innovation. $S$ has a lower limit. It has a weak impact on the participation intentions of the three parties. Further, necessary help and services from government will guarantee the three parties to reach the agreement of collaborative innovation.

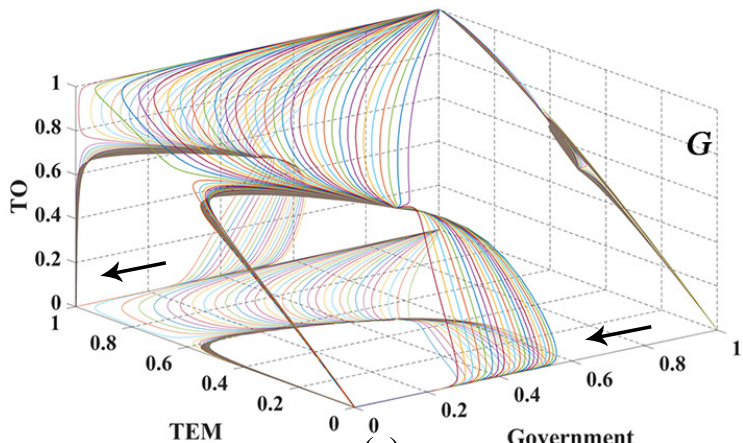

(a)

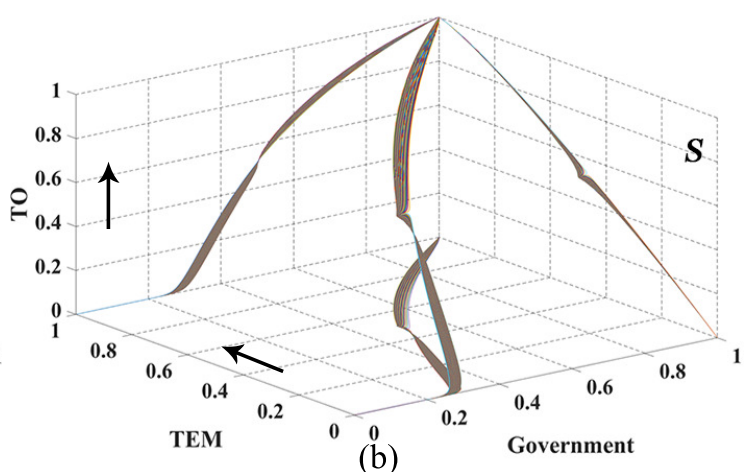

(b)

Figure 5. The influence of $G(\mathbf{a})$ and $S(\mathbf{b})$ on the evolutionary behaviors of the three parties; the arrow direction represents an increase in value.

In 2016, China launched its first NB-IoT scale commercial bureau in Fuzhou. Prior to that, Fuzhou municipal government signed cooperation agreements with Huawei and three typical operators successfully to build domestic NB-IoT application sample points and complete the deployment of base stations in an NB-IoT experimental unit. Besides, Fuzhou government set up a preparatory group, started to build a national NB-IoT open laboratory, studied and formulated corresponding work plans, and issued supportive policies to accelerate the development of the IoT industry. Government is not only the mentor of the IoT industry, but is also the planner of IoT infrastructure. It plays an important role in the competitiveness and innovation capacity in the industry. However, the situation varies from place to place. The government with low fiscal revenue may provide limited funding to deploy IoT base stations and appropriate basic facilities. For the government with high fiscal revenue, it has ability to further support the IoT industry. Such as reasonable and effective allocation of government funding, the establishment of IoT incubation base and industrial park, the popularization of IoT application demonstration area, and attention to R\&D of key technology and personnel training [48,49]. A variety of channels, e.g., mass innovation, special innovation, and transformation of scientific and technological achievements, will be guided correctly, to promote collaborative innovation of the IoT industry [49]. In addition, government is also a major proponent and participant in collaborative innovation. It is important but enough for the government to just provide necessary help and services, such as planning programming and approval process, for the innovative parties. Besides, the government, who has additional capability, can strengthen the support and advocacy of collaborative innovation, provide help for education and training, and accelerate and expand the flow among innovators, innovation platform, government, and other institutions, to promote the technical capacity in the IoT industry [50,51].

\subsection{Distribution}

The allocation process of social and economic resources not only affects innovation level and organizational behavior, but is also one of important factors to ensure the stable operation of industrial innovation platform [52-55]. In this paper, we use $\beta$ and $\delta$ as representatives of distribution. Figure 6a displays the influence of $\beta$ on the evolutionary behaviors of the three parties. The value of $\beta$ ranges from 0 to 1 with the step of 0.01 , and the arrow direction represents an increase in value. As $\beta$ increases, all of the three parties evolve from non-participation to participation to non-participation in collaborative innovation gradually. In front, top, and side views, when $\beta$ is low, TEM takes the lead 
to give up; when $\beta$ is high, TO gives up first. In both cases, the government gives up last. In order to determine the range of $\beta$ on condition all the three parties participate in collaborative innovation, we set $\beta$ as $0.49,0.50$, and 0.51 , respectively. Only when $\beta \geq 0.50$, can tripartite collaborative innovation take place in Figure 7a. When $\beta$ equals 0.49, TEM takes the lead in non-participation, then drives TO to give up, resulting in the government to abandon cooperation consequently. When $\beta$ equals 0.51 , all the three parties go in for collaborative innovation almost simultaneously. Their hesitation periods are shorter than those when $\beta$ equals 0.50 in Figure $7 \mathrm{~b}-\mathrm{d}$. These are all consistent with what is shown in Figure 6a. Figure $6 \mathrm{~b}$ shows the influence of $\delta$ on the evolutionary behaviors of the three parties. The value of $\delta$ ranges from 0 to 1 with the step of 0.01 , and the arrow direction represents an increase in value. As $\delta$ keeps increasing, all of the three parties evolve from non-participation to participation in collaborative innovation gradually. In front, top, and side views, when $\delta$ is low, TEM is the first to give up, leading TO with stronger participation intention to give up. Then, the government gives up too. When $\delta$ exceeds the critical value, TEM with stronger willingness will drive the government and TO with weaker willingness to participate in collaborative innovation. To sum up, $\beta$ can only be in a range, which is not less than 0.50. It can directly affect the participation intentions of TEM and TO in the evolutionary game process. In other words, in terms of profit distribution, if the stronger innovative party takes appropriate care of the weaker innovative party, it will accelerate both parties to reach the agreement of collaborative innovation. $\delta$ has a lower limit. It has a strong impact on the participation willingness of TEM and a weak impact on that of TO. Even if all government subsidies are distributed to TEM, the three parties are still in collaborative innovation consequently. Further, in the aspect of government subsidy distribution, that the government deliberately support the growth of weaker innovative party will promote the three parties to reach the consensus of collaborative innovation.

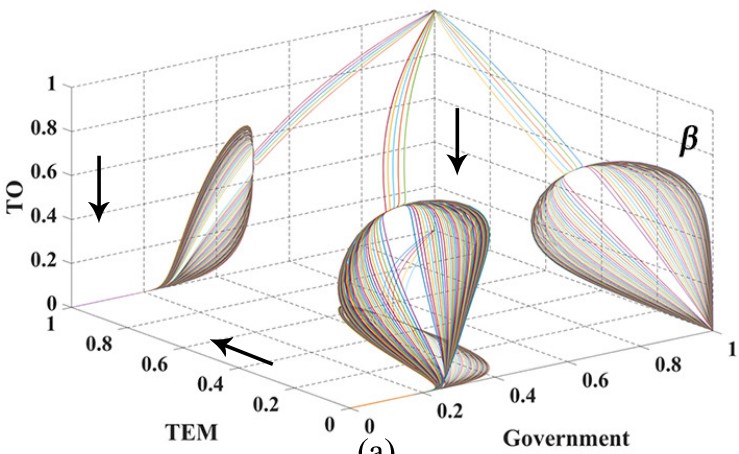

(a)

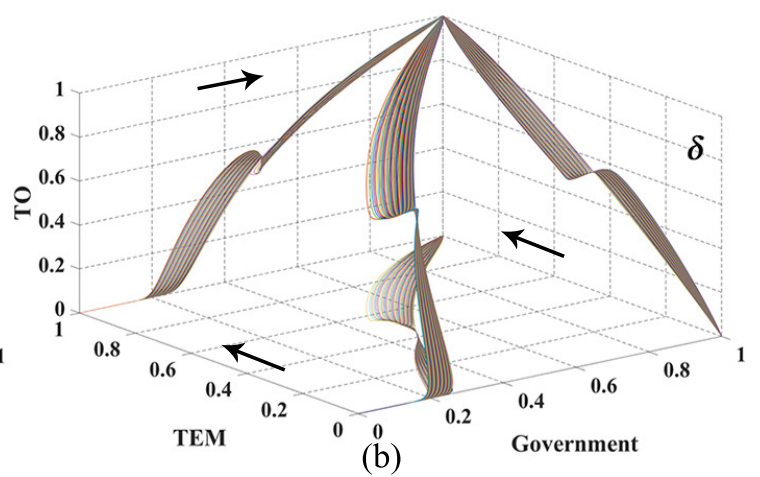

(b)

Figure 6. The influence of $\beta(\mathbf{a})$ and $\delta(\mathbf{b})$ on the evolutionary behaviors of the three parties; the arrow direction represents an increase in value.

In 2017, ZTE won the bidding for NB-IoT modules from China Telecom. China Telecom agreed to provide 30 yuan per piece for the winning manufacture, for the purpose of guiding the industry chain to migrate from GSM to NB-IoT, promoting the threshold of NB-IoT industry, and reducing costs. The IoT industry can be divided into perception layer, transmission layer, and application layer in the view of technology [11]. TO, as the information channel of IoT, occupy the leading position of the IoT industry by virtue of their previous technical experience and capital accumulation in China. It gives the whole IoT industry a conceptual shape of being large in the middle and narrow at both ends, like an olive. Therefore, in the R\&D process of IoT infrastructure, TO and government should cooperative and support the polar ends of the IoT industry to broaden the M2M market. As leader enterprises in the IoT industry, TO could promote the development of weak enterprises through profit distribution and other ways. It can help TO get rid of the revenue pressure of traditional business, explore the potential value of data pipelines, increase the vertical distribution, and promote the digital transformation themselves. It also improves the IoT industrial chain and promotes the linkage development of the IoT Industry. More importantly, early profit distribution needs to be clearly defined in advance [56]. It could not only facilitate the smooth process of collaborative innovation, but also improve the enthusiasm of 
weak enterprises to enliven the market. Meanwhile, government should be inclined to support small and medium-sized enterprises to improve the sustainable development of the IoT industry. It also should enhance the transparency and fairness of resource allocation and policy implementation to ensure the progress of collaborative innovation of the IoT industry under government leadership [57].

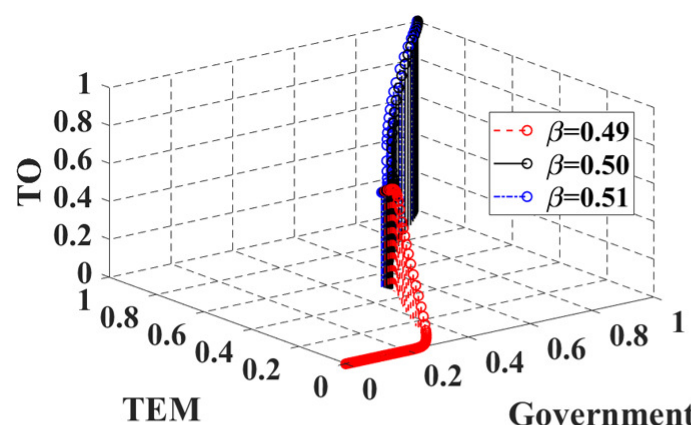

(a)

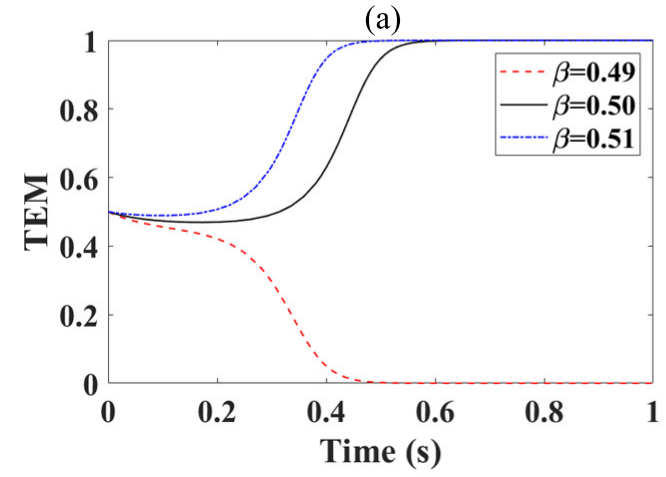

(c)

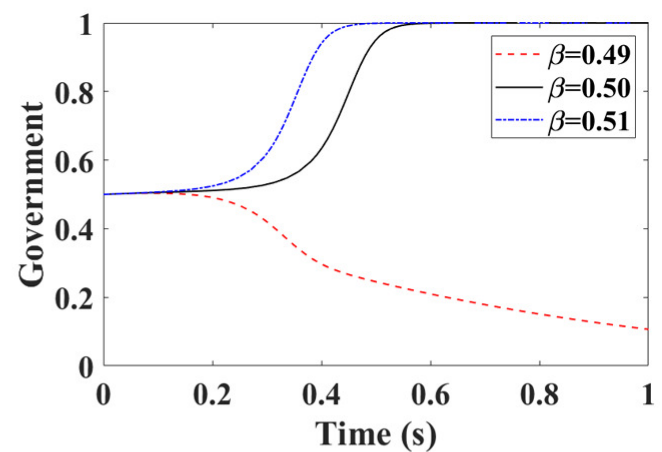

(b)

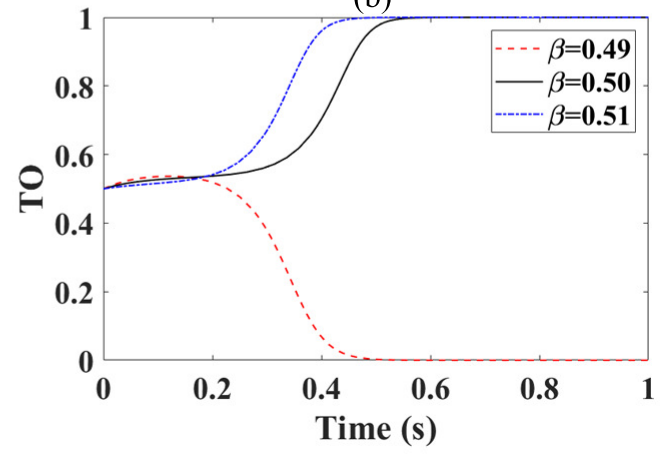

(d)

Figure 7. The influence of $\beta$ (a) on the evolutionary behaviors of the three parties, and the evolutionary trends of the government (b), TEM (c), and TO (d).

\subsection{Punishment}

In economic games, punishment often imposes a powerful price on those who break the rules [58]. Nowak commented that evolutionary models of punishment were based on underlying mechanisms such as indirect reciprocity, group selections, or network reciprocity. Punishment was an important factor to promote cooperation and improve cooperation level [59]. The government, employers, and partners can use punishment and other methods to achieve the desired goals [60]. In this paper, we use $G_{L}$ and $\theta+\rho$ as representatives of punishment. As we set $x=y=z=\alpha=0.5$, all of $\theta$ and $\rho$ in system Equation (13) are changed into the form of $\theta+\rho$. Figure 8a displays the influence of $G_{L}$ on the evolutionary behaviors of the three parties. The value of $G_{L}$ ranges from 0 to 20 with the step of 0.2 , and the arrow direction represents an increase in value. As $G_{L}$ keeps increasing, all of the three parties evolve from non-participation to participation in collaborative innovation gradually. In front, top, and side views, when $G_{L}$ is low, TEM and TO take the lead in non-participation, then the government follows. When $G_{L}$ is greater than the critical value, it has a similar impact on the participation willingness of all the three parties. Figure $8 \mathrm{~b}$ demonstrates the influence of $\theta+\rho$ on the evolutionary behaviors of the three parties. The value of $\theta+\rho$ ranges from 0 to 1 with the step of 0.01 , and the arrow direction represents an increase in value. When $\theta+\rho$ keeps increasing, all three parties evolve from non-participation to participation in collaborative innovation gradually. The graphs in front, top, and side views are similar as those in Figure 4a. Therefore, the $\theta+\rho$ has a more severe impact on the participation willingness of TEM and TO than that of the government. Moreover, it has a similar impact on the participation intentions of TEM and TO. To sum up, $G_{L}$ has a lower limit, in the evolutionary game process, and when $G_{L}$ is less than this lower limit, it can directly affect the participation intentions of TEM and TO. When $G_{L}$ is higher than the lower limit, the impact on the participation intentions 
of all the three parties is weak. $\theta+\rho$ also has a limit value. It can directly affect the participation intentions of TEM and TO, which subsequently drive the government to change. In other words, basic compensation of the government or innovative parties will guarantee the innovative parties to reach the agreement of collaborative innovation.

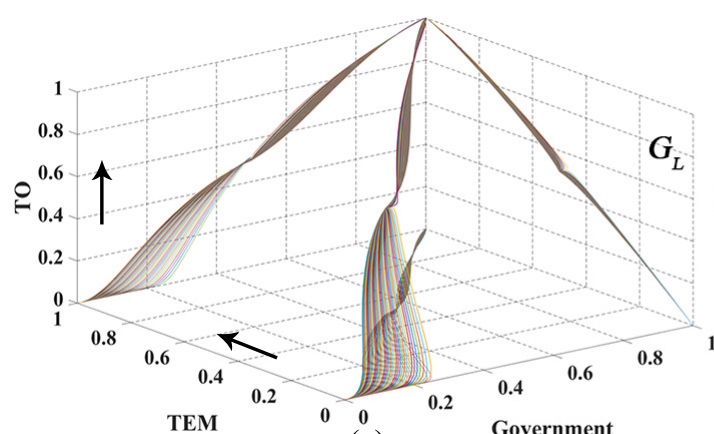

(a)

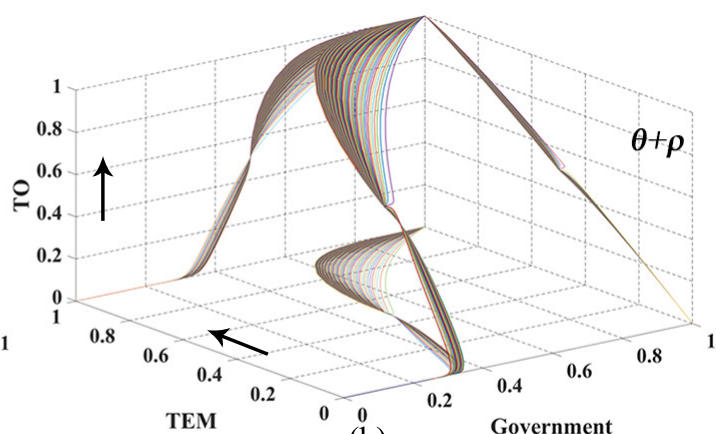

(b)

Figure 8. The influence of $G_{L}(\mathbf{a})$ and $\theta+\rho(\mathbf{b})$ on the evolutionary behaviors of the three parties; the arrow direction represents an increase in value.

Civilized human life is embedded in various institutions. Therefore, we need to form a relative perfect constraint system, establish corresponding rules and regulations, and issue corresponding industry norms. They can benefit $R \& D$ and application of IoT infrastructure components and the rapid development of IoT industry. Improving the effectiveness of punishment and enhancing communication and the intensity of identity construction can reduce the risk of betrayal [61]. Meanwhile, the principal of voluntary participation, reputation mechanism, and anti-corruption measures should be implemented to reduce the risk of second-level free-riding brought by punishment $[62,63]$. These are guarantees for collaborative innovation of the IoT industry under government leadership.

\section{Conclusions and Implications}

In this paper, we construct the evolutionary game model of the government, TEM, and TO, based on evolutionary game theory. Moreover, we make the analysis of the equilibrium stability, asymptotic stability, and evolutionary stable strategy of the three parties. Finally, we apply numerical simulation to discuss the eight factors and four mechanisms. The results are as follows.

- In terms of urban planning, both the increasing number of IoT base stations and the increasing expected social benefit will promote the three parties to reach the consensus of collaborative innovation. The increasing number of IoT base stations could reduce innovation risk and increase marginal revenue for the IoT industry subjects. The increasing expected social benefit could help the government with high fiscal revenue carry out those projects for beneficial economic cycle and time saving.

- In the aspect of government intervention, both government subsidy within reasonable range and necessary assistance will accelerate the three parties to reach the agreement of collaborative innovation. Government subsidy within reasonable range could help the government with low fiscal revenue provide limited funding to deploy IoT base stations and appropriate basic facilities or think about whether to hold on the project or not. Necessary assistance means government should treat the IoT industry with well-balanced tension and relaxation.

- In terms of distribution, either the stronger innovative party or the government takes appropriate care of the weaker innovative party will promote the three parties to reach the consensus of collaborative innovation. Supporting weak enterprises, like small and medium-sized enterprises, not only provides small business the opportunity to diversity the market, but also supports the industrial chain to promote the development of the IoT industry. 
- In the aspect of punishment, basic compensations of the government and innovative parties will guarantee the innovative parties to reach the agreement of collaborative innovation. Punishment is the basic safeguard to the development of IoT industry.

In fact, there are many problems to be solved before the deployment of IoT infrastructure for the government, as an initiator, and industrial subjects, as innovative parties. Evolutionary game model offers ideas for their collaborative innovation. First, it provides the government and innovative enterprises start point. That is, what to do and how to do it are their first concerned issues. Second, it gives different perspectives to the government according to its own situation, such as expected social benefit or government subsidy. Besides, the government receiving the tasks from the top may further consider about cost-performance ratio, like the ratio of expected social benefit and government subsidy. Third, it could offer theoretical basis for the government to streamline its administration and delegate authority, and to support the development of small and medium-sized enterprises. Fourth, it could provide implications for the IoT industry to reduce innovation risk and improve the industrial chain.

The IoT industry, which is at an important time in its development and deployment, should be taken as the main driver of sustainable economic development. It can be combined with Big Data and Cloud Computing to lay a foundation for the arrival of 5G and build the IoT industry ecosystem and smart cities with international competitiveness and influence. Collaborative innovation can fully utilize the strengths of others, integrate complementary resources, and accelerate the application and industrialization of technologies. It is the only way to build an innovation-oriented country. Therefore, government and IoT industry main bodies should carry out top-level design in the combination of the characteristics of the new generation of information technology industry. Clarifying the concept of innovation-driven development and planning the innovation and development strategy of the IoT industry are of importance. The organizational structure of collaborative innovation system with clear functions should be formed and collaborative innovation situation with multi-subject cooperation should be open. At the same time, an interconnection mechanism between collaborative innovation entities should be established to realize the deep integration of various innovation resources. Systems and mechanisms that combine government and market should be further improved. The market plays the role of basic regulation and the government plays the role of guidance, regulation, and support. Government can provide preferential policies and measures to realize IoT industrial clusters. It also can create a good policy, experiment, and platform environment for collaborative innovation of the IoT industry to promote its orderly and steady development. Dominant firms could expand the application of IoT in new technology and business, actively guide integrated innovation with vertical industries, and carry out collaborative innovation of joint technology. Meanwhile, they, together with government, could lead a group of small and medium-sized enterprises, who are mainly driven by scientific and technological innovation, to enrich and improve the industrial chain. Further, they could attach importance to the innovation needs of small and medium-sized enterprises, and encourage these enterprises to carry out collaborative innovation based on their common innovation goals. Establishing and improving intellectual property laws and regulations, increasing penalties for negative cooperation and subsidy fraud, and creating a positive atmosphere for promoting collaborative innovation of the IoT industry can accelerate the pace of IoT infrastructure innovation.

This work mainly discusses how to reach an agreement on collaborative innovation of the IoT industry under government leadership. However, it has some limitations due to model and data sources. Only the main factors affecting collaborative innovation are analyzed and simplified. Additionally, we only conduct research in the context of China due to particular factors including huge population, relatively perfect supply chain and industrial chain, and broad market of IoT in the future. The future work will carry out research on the similarities and differences of collaborative innovation in IoT infrastructure construction between Chinese and foreign IoT industry entities.

Author Contributions: J.H. conceived and designed the research; B.L. supervised and revised the whole paper. All authors have read and agreed to the published version of the manuscript. 
Funding: This research was funded by "The Key Program of National Social Science Foundation of China" grant number 19FGLA001.

Conflicts of Interest: The authors declare no conflict of interest.

\section{References}

1. Miorandi, D.; Sicari, S.; De Pellegrini, F.; Chlamtac, I. Internet of Things: Vision, applications and research challenges. Ad Hoc Netw. 2012, 10, 1497-1516. [CrossRef]

2. Tan, L.; Wang, N. Future internet: The Internet of Things. In Proceedings of the ICACTE, Chengdu, China, 20-22 August 2010; pp. 376-380.

3. Kamble, S.S.; Gunasekaran, A.; Parekh, H.; Joshi, S. Modeling the Internet of Things adoption barriers in food retail supply chains. J. Retail. Consum. Serv. 2019, 48, 154-168. [CrossRef]

4. Xu, L.D.; He, W.; Li, S. Internet of Things in industries: A survey. IEEE Trans. Ind. Inform. 2014, 10, $2233-2243$. [CrossRef]

5. Zanella, A.; Bui, N.; Castellani, A.; Vangelista, L.; Zorzi, M. Internet of Things for smart cities. IEEE Internet Things J. 2014, 1, 22-32. [CrossRef]

6. Botta, A.; de Donato, W.; Persico, V.; Pescape, A. Integration of Cloud Computing and Internet of Things: A survey. Future Gener. Comput. Syst. 2016, 56, 684-700. [CrossRef]

7. Chen, M.; Mao, S.; Liu, Y. Big Data: A Survey. Mob. Netw. Appl. 2014, 19, 171-209. [CrossRef]

8. Li, L. China's manufacturing locus in 2025: With a comparison of "Made-in-China 2025" and "Industry 4.0". Technol. Forecast. Soc. Chang. 2018, 135, 66-74. [CrossRef]

9. Kagermann, H.; Wahlster, W.; Helbig, J. Recommendations for Implementing the Strategic Initiative Industrie 4.0: Final Report of the Industrie 4.0 Working Group; Acatech: Munich, Germany, 2013.

10. Weiser, M. The computer for the 21st century. Sci. Am. 1991, 265, 94-104. [CrossRef]

11. Yang, Z.; Zou, G. Research on strengthening independent innovation capability of Internet of Things enterprises: A case study on the R\&D of 2-dimentional barcode technology in Newland Computer Co. Ltd. In Proceedings of the PICMET, Portland, OR, USA, 31 July-4 August 2011; pp. 1-7.

12. Haken, H. Synergetics of brain function. Int. J. Psychophysiol. 2006, 60, 110-124. [CrossRef]

13. Bresciani, S.; Ferraris, A.; Del Giudice, M. The management of organizational ambidexterity through alliances in a new context of analysis: Internet of Things (IoT) smart city projects. Technol. Forecast. Soc. Chang. 2018, 136, 331-338. [CrossRef]

14. Nieto, M.J.; Santamaría, L. The importance of diverse collaborative networks for the novelty of product innovation. Technovation 2007, 27, 367-377. [CrossRef]

15. Bengtsson, M.; Kock, S. "Coopetition" in business networks-to cooperate and compete simultaneously. Ind. Mark. Manag. 2000, 29, 411-426. [CrossRef]

16. Mihailescu, B.; Svasta, P.; Marghescu, C.; Borcea, A. Clusters as a catalyst for developing human resources for the Internet of Things industry. In Proceedings of the ISSE, Eger, Hungary, 6-10 May 2015; pp. 522-525.

17. Qi, Y.; Wang, J. Empirical research on collaborative innovation of strategic emerging industry based on system science. In Proceedings of the ICSSSM, Kunming, China, 24-26 June 2016; pp. 1-6.

18. Chi, M.; Wang, W.; Lu, X.; George, J.F. Antecedents and outcomes of collaborative innovation capabilities on the platform collaboration environment. Int. J. Inf. Manag. 2018, 43, 273-283. [CrossRef]

19. Fernandez-Esquinas, M.; Pinto, H.; Yruela, M.P.; Pereira, T.S. Tracing the flows of knowledge transfer: Latent dimensions and determinants of university-industry interactions in peripheral innovation systems. Technol. Forecast. Soc. Chang. 2016, 113, 266-279. [CrossRef]

20. Lv, B.; Qi, X. Research on partner combination selection of the supply chain collaborative product innovation based on product innovative resources. Comput. Ind. Eng. 2019, 128, 245-253. [CrossRef]

21. Nilssen, M. To the smart city and beyond? Developing a typology of smart urban innovation. Technol. Forecast. Soc. Chang. 2019, 142, 98-104. [CrossRef]

22. Zhang, X.; Wang, F. Research on the uncertainty decision model of the regional collaborative innovation system based on an improved ant colony algorithm. Sci. Program. 2016, 2016. [CrossRef]

23. Zhao, J.; Wu, G.; Xi, X.; Na, Q.; Liu, W. How collaborative innovation system in a knowledge-intensive competitive alliance evolves? An empirical study on China, Korea and Germany. Technol. Forecast. Soc. Chang. 2018, 137, 128-146. [CrossRef] 
24. Lu, T.; Zhou, J. Research on multiple factor linkages in innovation system of IoT industry in China. Chin. Soft Sci. 2011, 3, 33-45.

25. Jiao, Y.; Shen, Z.; Hu, Q. Research on partnership of strategic emerging industry collaborative innovative network under different dominant roles-A case study on Chinese IoT. R\&D Manag. 2015, 27, 60-74.

26. Shao, Y.; Lv, W. A symbiosis theory based study on collaborative innovation of the Internet of Things in China led by telecom carriers. Chin. J. Manag. 2016, 13, 239-247.

27. Yu, X.; Nguyen, B.; Chen, Y. Internet of Things capability and alliance: Entrepreneurial orientation, market orientation and product and process innovation. Internet Res. 2016, 26, 402-434. [CrossRef]

28. Friedman, D. Evolutionary games in economics. Econometrica 1991, 59, 637-666. [CrossRef]

29. Hofbauer, J.; Sigmund, K. Evolutionary games dynamics. Bull. Am. Math. Soc. 2003, 40, 479-519. [CrossRef]

30. Tong, X.; Yu, L. Evolutionary game analysis on the co-opetition of enterprise innovation in hi-tech industry cluster. In Proceedings of the ICMSE, Harbin, China, 17-19 July 2013; pp. 1955-1961.

31. Liu, W.; Yang, J. The evolutionary game theoretic analysis for sustainable cooperation relationship of collaborative innovation network in strategic emerging industries. Sustainability 2018, 10, 4585. [CrossRef]

32. Palattella, M.R.; Dohler, M.; Grieco, A.; Rizzo, G.; Torsner, J.; Engel, T.; Ladid, L. Internet of Things in the 5G era: Enablers, architecture, and business models. IEEE J. Sel. Areas Commun. 2016, 34, 510-527. [CrossRef]

33. Yeon, S.; Jun, H. Issues and implementation strategies of the IoT (Internet of Things) industry. In Proceedings of the IMIS, Fukuoka, Japan, 6-8 July 2016; pp. 503-508.

34. Wang, J. Innovation and government intervention: A comparison of Singapore and Hong Kong. Res. Policy 2018, 47, 399-412. [CrossRef]

35. Watkins, A.; Papaioannou, T.; Mugwagwa, J.; Kale, D. National innovation systems and the intermediary role of industry associations in building institutional capacities for innovation in developing countries: A critical review of the literature. Res. Policy 2015, 44, 1407-1418. [CrossRef]

36. Gintis, H. Game Theory Evolving: A Problem-Centered Introduction to Modeling Strategic Interaction, 2nd ed.; Princeton University: Princeton, NJ, USA, 2009; pp. 255-265.

37. Ahern, J.; Cilliers, S.; Niemela, J. The concept of ecosystem services in adaptive urban planning and design: A framework for supporting innovation. Landsc. Urban Plan. 2014, 125, 254-259. [CrossRef]

38. Babar, M.; Arif, F. Smart urban planning using Big Data analytics to contend with the interoperability in Internet of Things. Future Gener. Comput. Syst. 2017, 77, 65-76. [CrossRef]

39. Newton, P.W. Innovation for a sustainable low carbon built environment. In Proceedings of the IHBE, Sydney, Australia, 17-18 November 2016; pp. 16-32.

40. Jin, J.; Gubbi, J.; Marusic, S.; Palaniswami, M. An information framework for creating a smart city through Internet of Things. IEEE Internet Things J. 2014, 1, 112-121. [CrossRef]

41. Gubbi, J.; Buyya, R.; Marusic, S.; Palaniswami, M. Internet of Things (IoT): A vision, architectural elements, and future directions. Future Gener. Comput. Syst. 2013, 29, 1645-1660. [CrossRef]

42. Jia, M.; Komeily, A.; Wang, Y.; Srinivasan, R.S. Adopting Internet of Things for the development of smart buildings: A review of enabling technologies and applications. Autom. Constr. 2019, 101, 111-126. [CrossRef]

43. Rathore, M.M.; Ahmad, A.; Paul, A.; Rho, S. Urban planning and building smart cities based on the Internet of Things using Big Data analytics. Comput. Netw. 2016, 101, 63-80. [CrossRef]

44. Silva, B.N.; Khan, M.; Jung, C.; Seo, J.; Muhammad, D.; Han, J.; Yoon, Y.; Han, K. Urban planning and smart city decision management empowered by real-time data processing using Big Data analytics. Sensors 2018, 18, 2994. [CrossRef] [PubMed]

45. Gao, P. Government in the catching-up of technology innovation: Case of administrative intervention in China. Technol. Forecast. Soc. Chang. 2015, 96, 4-14. [CrossRef]

46. Yin, S.; Li, B.Z.; Xing, Z.Y. The governance mechanism of the building material industry (BMI) in transformation to green BMI: The perspective of green building. Sci. Total Environ. 2019, 677, 19-33. [CrossRef]

47. Droste, N.; Hansjurgens, B.; Kuikman, P.; Otter, N.; Antikainen, R.; Leskinen, P.; Pitkanen, K.; Saikku, L.; Loiseau, E.; Thomsen, M. Steering innovations towards a green economy: Understanding government intervention. J. Clean. Prod. 2016, 135, 426-434. [CrossRef]

48. Hong, J.; Feng, B.; Wu, Y.; Wang, L. Do government grants promote innovation efficiency in China's high-tech industries? Technovation 2016, 57-58, 4-13. [CrossRef] 
49. Li, X.; Gao, Y.; Zhao, H. Comparative research on the IoT industry competitiveness of eastern, central and western China. In Proceedings of the IEEE GSIS, Leicester, UK, 18-20 August 2015; pp. 187-193.

50. Zhang, L.; Wu, B. Farmer innovation system and government intervention: An empirical study of straw utilization technology development and diffusion in China. J. Clean. Prod. 2018, 188, 698-707. [CrossRef]

51. Joo, H.; Seo, Y.; Min, H. Examining the effects of government intervention on the firm's environmental and technological innovation capabilities and export performance. Int. J. Prod. Res. 2018, 56, 6090-6111. [CrossRef]

52. Li, D.; Lin, J.; Cui, W.; Qian, Y. The trade-off between knowledge exploration and exploitation in technological innovation. J. Knowl. Manag. 2018, 22, 781-801. [CrossRef]

53. Wang, L.; Tan, Q.; Lu, X. Profit distribution mechanism of industrial innovation platform research. In Proceedings of the IEEE GSIS, Stockholm, Sweden, 8-11 August 2017; pp. 15-18.

54. Wu, J.; Ma, L.; Yang, Y. Innovation in the Chinese public sector: Typology and distribution. Public Adm. 2013, 91, 347-365. [CrossRef]

55. Zeppini, P.; van den Bergh, J.C.J.M. Optimal diversity in investments with recombinant innovation. Struct. Chang. Econ. Dyn. 2013, 24, 141-156. [CrossRef]

56. Cao, J.; Shen, M.; Fan, D. The game analysis on interest distribution of industry-university-research cooperative technology Innovation. In Proceeding of the ICMSEM, Rome, Italy, 13-15 September 2011; pp. 1081-1087.

57. Hou, Q.; Hu, M.; Yuan, Y. Corporate innovation and political connections in Chinese listed firms. Pac.-Basin Financ. J. 2017, 46, 158-176. [CrossRef]

58. Perc, M.; Jordan, J.J.; Rand, D.G.; Wang, Z.; Boccaletti, S.; Szolnoki, A. Statistical physics of human cooperation. Phys. Rep.-Rev. Sec. Phys. Lett. 2017, 687, 1-51. [CrossRef]

59. Nowak, M.A. Five rules for the evolution of cooperation. Science 2006, 314, 1560-1563. [CrossRef]

60. Parks, C.D.; Joireman, J.; Van Lange, P.A.M. Cooperation, trust, and antagonism: How public goods are promoted. Psychol. Sci. Public Interest 2013, 14, 119-165. [CrossRef]

61. Weng, Q.; Carlsson, F. Cooperation in teams: The role of identity, punishment, and endowment distribution. J. Public Econ. 2015, 126, 25-38. [CrossRef]

62. dos Santos, M.; Rankin, D.J.; Wedekind, C. The evolution of punishment through reputation. Proc. R. Soc. $B$ Biol. Sci. 2011, 278, 371-377. [CrossRef]

63. Huang, F.; Chen, X.; Wang, L. Evolution of cooperation in a hierarchical society with corruption control. J. Theor. Biol. 2018, 449, 60-72. [CrossRef] [PubMed]

(C) 2020 by the authors. Licensee MDPI, Basel, Switzerland. This article is an open access article distributed under the terms and conditions of the Creative Commons Attribution (CC BY) license (http:/ / creativecommons.org/licenses/by/4.0/). 